\title{
Increased Robustness of Single-Molecule Counting with Microfluidics, Digital Isothermal Amplification, and a Mobile Phone versus Real-Time Kinetic Measurements
}

\author{
David A. Selck, ${ }^{\text {II }}$ Mikhail A. Karymov, ${ }^{\text {II }}$ Bing Sun, ${ }^{\text {II }}$ and Rustem F. Ismagilov* \\ Division of Chemistry and Chemical Engineering, California Institute of Technology, 1200 East California Boulevard, Pasadena, \\ California 91125, United States
}

Supporting Information

\begin{abstract}
Quantitative bioanalytical measurements are commonly performed in a kinetic format and are known to not be robust to perturbation that affects the kinetics itself or the measurement of kinetics. We hypothesized that the same measurements performed in a "digital" (single-molecule) format would show increased robustness to such perturbations. Here, we investigated the robustness of an amplification reaction (reverse-transcription loopmediated amplification, RT-LAMP) in the context of fluctuations in temperature and time when this reaction is used for quantitative measurements

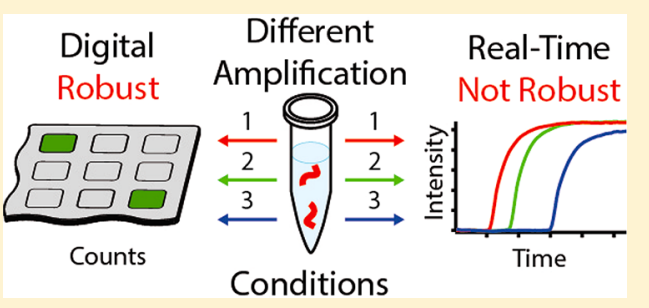
of HIV-1 RNA molecules under limited-resource settings (LRS). The digital format that counts molecules using dRT-LAMP chemistry detected a 2-fold change in concentration of HIV-1 RNA despite a 6 ${ }^{\circ} \mathrm{C}$ temperature variation $\left(p\right.$-value $\left.=6.7 \times 10^{-7}\right)$, whereas the traditional kinetic (real-time) format did not $(p$-value $=0.25)$. Digital analysis was also robust to a $20 \mathrm{~min}$ change in reaction time, to poor imaging conditions obtained with a consumer cellphone camera, and to automated cloud-based processing of these images $\left(R^{2}=0.9997\right.$ vs true counts over a 100 -fold dynamic range). Fluorescent output of multiplexed PCR amplification could also be imaged with the cell phone camera using flash as the excitation source. Many nonlinear amplification schemes based on organic, inorganic, and biochemical reactions have been developed, but their robustness is not well understood. This work implies that these chemistries may be significantly more robust in the digital, rather than kinetic, format. It also calls for theoretical studies to predict robustness of these chemistries and, more generally, to design robust reaction architectures. The SlipChip that we used here and other digital microfluidic technologies already exist to enable testing of these predictions. Such work may lead to identification or creation of robust amplification chemistries that enable rapid and precise quantitative molecular measurements under LRS. Furthermore, it may provide more general principles describing robustness of chemical and biological networks in digital formats.
\end{abstract}

\begin{abstract}
A wide range of nonlinear and especially autocatalytic - chemical amplification schemes is being developed and studied using organic, inorganic, and biochemical reactions. ${ }^{1-12}$ These studies are motivated both by understanding of signal transduction in natural systems and by opportunities for rapid, ultrasensitive detection and quantification of analyte molecules. In natural systems, networks of biochemical reactions crucial for the function of living organisms are robust to intrinsic and extrinsic fluctuations, to environmental changes, and even to some perturbations not likely to be previously encountered by the organism. ${ }^{13-16}$ However, robustness of synthetic nonlinear chemical amplification schemes is not yet fully understood: despite tremendous progress in experimental and theoretical studies of nonlinear chemical dynamics, ${ }^{11}$ it is not clear which specific reactions or general reaction architectures are robust to which perturbations. Robustness becomes an especially relevant property of a nonlinear chemical amplification scheme when the amplification is used for quantitative analytical measurements. Quantitative measurements of biomolecules are essential for addressing a range of societal problems in human health, food and water safety, ${ }^{17,18}$ environmental monitoring, ${ }^{19}$ and biosecurity. ${ }^{20}$
\end{abstract}

Typically, kinetic assays are used for quantitative measurements. ${ }^{21}$ These assays are performed through monitoring the progress of a reaction at a single time point in an end-point measurement or at multiple time points in a real-time measurement. Because both extent of reaction (e.g., a concentration measured by an optical imaging method) and time must be measured in kinetic assays, one would expect the results of such assays to be sensitive to changes in reaction conditions and to fluctuations in the performance of the readout instrument. For a linear amplification scheme in a kinetic format, a temperature change leading to a 50\% change in the rate constant would lead to a $50 \%$ error in quantification. One would expect an autocatalytic amplification scheme to be especially sensitive to these fluctuations because small kinetic perturbations would become exponentially compounded: a $50 \%$ change in the rate constant could lead to over a $400000 \%$ error in quantification (see Supporting Information online). This problem is avoided in real-time polymerase chain reactions

Received: September 23, 2013

Accepted: October 17, 2013

Published: November 7, 2013 
a) Quantitative measurements -- under ideal conditions

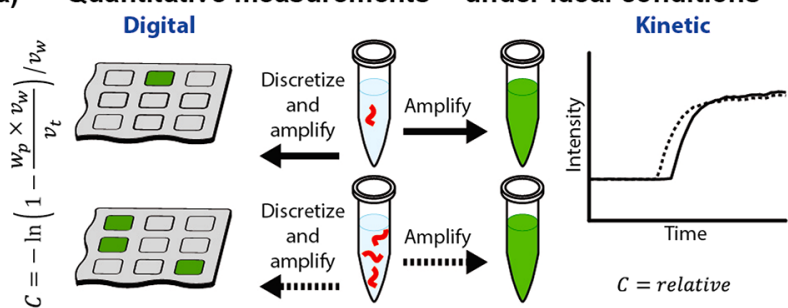

c)

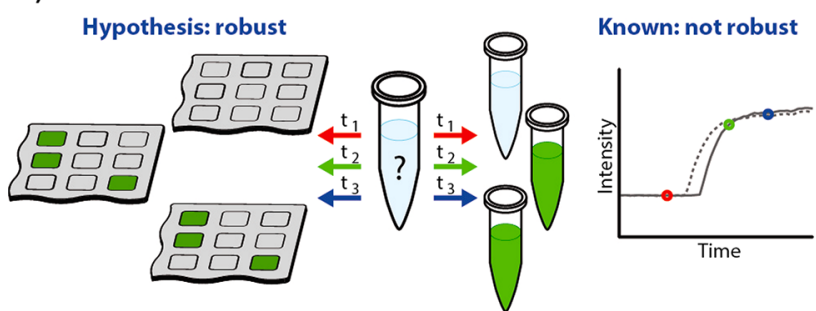

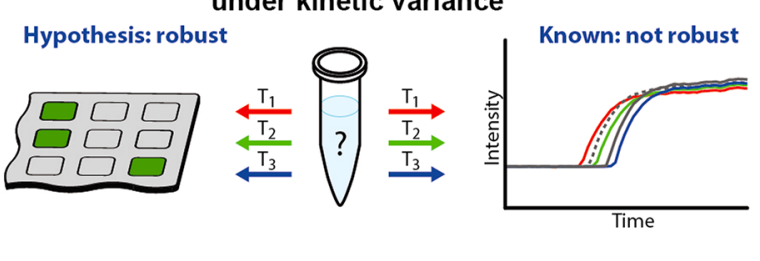

d)

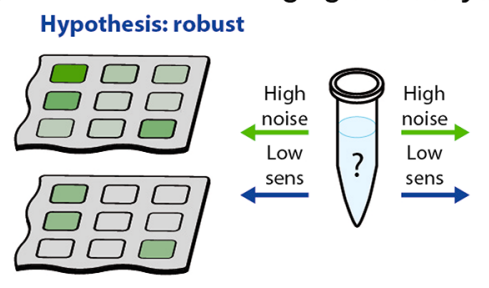

under imaging and analysis variance

Known: not robust

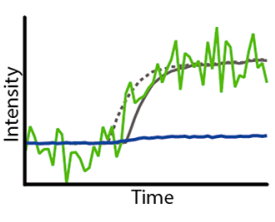

Figure 1. Robustness of quantification in digital vs kinetic formats. Cartoons for the curves in the kinetic format are drawn to resemble a specific case of real-time nucleic acid amplification. (a) An illustration comparing digital and kinetic formats under ideal conditions. In a digital format, individual molecules are separated into compartments and amplified, requiring only an end-point readout. The original concentration $(C)$ of the analyte can be calculated by the equation on the left (where $w_{\mathrm{p}}=$ the number of positive wells, $v_{\mathrm{t}}=$ the total device volume, and $v_{\mathrm{w}}=$ the volume of each well). In a kinetic format, the analyte is amplified in a bulk culture and the progress of amplification, measured as intensity, is monitored as a function of time. The original concentration is determined by comparing the reaction trace to standard curves from solutions of known concentration. (b) An illustration of the effects of kinetic variation (shown as differences in amplification temperature) in digital and real-time formats. In a digital format, we hypothesized that variance in the kinetic rate of amplification would not affect the end-point readout. In a real-time format, the kinetic rate determines the reaction curve and thus the relative concentration; therefore, it is known to be not robust. (c) An illustration of the effects of time variance (shown as readout time) in digital and real-time formats. Since digital requires only end-point readout, we hypothesized that exact knowledge of time would not be required and the output should be robust to variation in reaction time beyond the optimal reaction time. In a realtime format, precise knowledge of time and sufficient time points are required in order to accurately quantify concentration; therefore, it is known to be not robust to variation in reaction time. (d) An illustration of the effects of imaging in digital and real-time formats. In a digital format, one only needs to be able to distinguish a positive from a negative signal, and therefore, we hypothesized that imaging conditions with either increased noise or decreased sensitivity would not affect the measurement or data analysis. In a real-time format, imaging conditions with increased noise or decreased sensitivity can affect quantitative ability by producing reaction traces that cannot be compared to standards; therefore, it is known to be not robust to variation in imaging conditions.

(qPCR) because temperature cycling "gates" the amplification process: to a first approximation, as long as amplification kinetics remain fast enough to complete one doubling of the nucleic acid target within one PCR cycle, changes in the rate of this amplification should not affect the result. ${ }^{22,23}$ The majority of biological and chemical amplification strategies are isothermal and therefore cannot take advantage of this "gating".

Any study of robustness must be defined within a particular context that sets the type and magnitude of perturbations that might affect the system being studied. We chose the context of quantitative measurements performed under limited-resource settings (LRS). These settings lack sophisticated equipment and expertise ${ }^{24}$ and span both the developing and the developed world (e.g., at-home or field testing). Under these settings, one may expect variation of temperature of several degrees Celsius, imaging performed with nonquantitative consumer electronic devices such as cell phones, and variations in assay time due to operator error. While a number of robust qualitative tests, such as home pregnancy tests, are already compatible with LRS, suitable quantitative assays are needed. For instance, a high-quality, inexpensive, rapid HIV-1 viral load assay for LRS would enable infant diagnosis and monitoring of the emergence of drug resistance during therapy. ${ }^{25}$ While clinically used HIV-1 viral load assays measure HIV-1 RNA with relatively high precision $(<30 \% \mathrm{CV}){ }^{26}$ at least 3 -fold change in viral load is considered to be clinically significant. ${ }^{27}$
Autocatalytic amplification schemes ${ }^{1-12}$ are attractive for these settings; in principle, they could convert the presence of a few molecules to a large signal observed and quantified by eye or an inexpensive optical setup. In practice, among other factors, their use under LRS is hampered by the lack of robustness to experimental perturbations.

We hypothesized that autocatalytic, exponential amplification would be more robust to variations in temperature, time, and imaging quality in a "digital" format rather than a kinetic, realtime format (Figure 1). In a typical digital analysis, ${ }^{28-31}$ a sample is separated into discrete volumes such that not all volumes contain a molecule of interest (Figure 1a). After amplification, only those volumes that contained one or more molecules of interest produce a fluorescent signal. This information can be used to calculate the concentration of the target molecule using Poisson statistics. We hypothesized that, since for quantification, these "digital" methods require simply counting "positive" and "negative" reaction wells and thus do not require knowledge of time, reaction kinetics, or precise measurement of reaction progress, the results obtained by digital methods would not be affected strongly by temperature fluctuations (Figure 1b) or reaction time (Figure 1c) and should not require precise imaging instruments or very sophisticated analysis algorithms (Figure 1d). Therefore, we also hypothesized that as long as the amplification chemistry produces a specific and sufficiently bright optical signal, 
semiquantitative imaging devices such as cell phone cameras $^{32-34}$ should be able to detect positive signals and differentiate them from negative signals, thereby providing quantitative information. This hypothesis relies on a significant assumption: it can become true only if initiation of amplification reactions from single molecules is robust to these perturbations. Digital PCR is now commercially available and is used for a number of research applications. The limits of its robustness remain to be investigated; it is not obvious that it would be robust because results of digital PCR are known to be affected by experimental conditions. ${ }^{35}$ Such work is outside the scope of the present paper, as we are focusing on the more general question of robustness of isothermal amplification strategies, which also has not been investigated in this context.

\section{EXPERIMENTAL SECTION}

Real-Time Reverse-Transcription Loop-Mediated Amplification (RT-LAMP) of HIV-1 RNA. For two-step RTLAMP, a first solution $(20 \mu \mathrm{L})$, containing $10 \mu \mathrm{L}$ of RM, $1 \mu \mathrm{L}$ of bovine serum albumin (BSA), $0.5 \mu \mathrm{L}$ of EXPRESS SYBR GreenER RT module, $0.5 \mu \mathrm{L}$ of BIP primer $(10 \mu \mathrm{M})$, various amounts of template, and nuclease-free water, was first incubated at $50{ }^{\circ} \mathrm{C}$ for $10 \mathrm{~min}$ and then mixed with a second solution $(20 \mu \mathrm{L})$, containing $10 \mu \mathrm{L}$ of RM, $1 \mu \mathrm{L}$ of BSA, $2 \mu \mathrm{L}$ of EM, 1 or $2 \mu \mathrm{L}$ of $\mathrm{FD}, 2 \mu \mathrm{L}$ of other primer mixture, $1 \mu \mathrm{L}$ of Hybridase Thermostable RNase $\mathrm{H}$, and nuclease-free water. The $40 \mu \mathrm{L}$ mixture was split into 4 aliquots and loaded onto an Eco real-time PCR machine. For one-step RT-LAMP, a $40 \mu \mathrm{L}$ RT-LAMP mix contained the following: $20 \mu \mathrm{L}$ of RM, $2 \mu \mathrm{L}$ of BSA $(20 \mathrm{mg} / \mathrm{mL}), 2 \mu \mathrm{L}$ of EM, $2 \mu \mathrm{L}$ of FD, $2 \mu \mathrm{L}$ of primer mixture, a various amount of template solution, and nucleasefree water. The mixture was split into 4 aliquots and loaded onto the Eco real-time PCR machine. Data analysis was performed using Eco software (see details in Supporting Information online).

Two-Step dRT-LAMP of HIV-1 RNA on SlipChip. The procedures used to perform two-step dRT-LAMP on SlipChip were described in a previous publication. ${ }^{36} \mathrm{~A}$ first solution (equivalent to the one described above) was loaded onto a SlipChip device and incubated at $50{ }^{\circ} \mathrm{C}$ for $10 \mathrm{~min}$, and then a second solution (equivalent to the one described above) was loaded onto the same device and mixed with the first solution. The entire filled device was incubated at various temperatures $\left(57,60\right.$, or $\left.63{ }^{\circ} \mathrm{C}\right)$ for $60 \mathrm{~min}$.

Multiplexed PCR Amplification on SlipChip. The PCR mixture used for amplification of Staphylococcus aureus genomic DNA on a multiplexed SlipChip contained the following: $10 \mu \mathrm{L}$ of $2 \mathrm{X}$ SsoFast Evagreen SuperMix (BioRad, CA), $1 \mu \mathrm{L}$ of BSA $(20 \mathrm{mg} / \mathrm{mL}), 1 \mu \mathrm{L}$ of $1 \mathrm{ng} / \mu \mathrm{L}$ gDNA, $0.5 \mu \mathrm{L}$ of SYBR Green $(10 \times)$, and $7.5 \mu \mathrm{L}$ of nuclease-free water. Primers were preloaded onto the chip using a previously described technique (see details in Supporting Information online). The PCR amplification was performed with an initial $95{ }^{\circ} \mathrm{C}$ step for $5 \mathrm{~min}$ and then followed by 40 cycles of: (i) $1 \mathrm{~min}$ at $95^{\circ} \mathrm{C}$, (ii) $30 \mathrm{~s}$ at $55^{\circ} \mathrm{C}$, and (iii) $45 \mathrm{~s}$ at $72{ }^{\circ} \mathrm{C}$. An additional $5 \mathrm{~min}$ at $72{ }^{\circ} \mathrm{C}$ was performed to allow thorough dsDNA extension.

Cell Phone Camera Setup and Settings. A Nokia 808 Pureview cell phone was used to image and count microwells containing the amplification product. The Nokia 808 features 41-megapixel sensor with a pixel size of $1.4 \mu \mathrm{m}$. The camera uses pixel oversampling technology, which combines multiple pixels to increase the sensitivity of each individual pixel in the final image. Using a commercially available $0.67 \times$ objective, we were able to obtain images at a distance of $6.5 \mathrm{~cm}$, thus further improving imaging sensitivity. A set of two filters was used to both excite and detect fluorescence. Two excitation filters (FD1B) were stacked and attached in front of the camera flash. For fluorescence detection, two 5CGA-530 long-pass filters were inserted into a magnetically mounted lens (see Supporting Information online for more details).

Cell phone imaging of dRT-LAMP devices was performed with the devices tilted at $\sim 10$ degrees relative to the cell phone plane to prevent direct reflection of the flash into the lens. All images were taken using the standard cell phone camera application. The white balance was set to automatic, the ISO was set at 800 , the exposure value was set at +2 , the focus mode was set to "close-up", and the resolution was adjusted to $8 \mathrm{MP}$.

Cell phone imaging of multiplexed PCR devices was performed by imaging the devices in a shoebox painted black. The white balance was set to automatic, the ISO was set at 1600 , the exposure value was set at +4 , the focus mode was set to "close-up", and the resolution was adjusted to $8 \mathrm{MP}$. Images were processed using a free Fiji image processing package available on the Internet (see Supporting Information online for details of the procedure).

Cloud-Based Automatic Analysis. The Symbian software on which the Nokia 808 cell phone is based can access Skydrive, a cloud-based storage service. This service can automatically upload images to the cloud directly after imaging, without any user intervention. Here, we used a central computer with a custom Labview program to process all the uploaded files automatically. The process detailing the image analysis, as well as a video demonstrating that a minimally trained user can use the approach described in this paper, can be found in the Supporting Information online.

\section{RESULTS AND DISCUSSION}

To test these hypotheses, we selected HIV-1 RNA as a target molecule and selected isothermal digital reverse transcriptionloop-mediated amplification (dRT-LAMP) as the amplification chemistry. We chose LAMP amplification chemistry for three reasons: (i) When performed with a qualitative readout, in at least one example, it is known to tolerate a number of perturbations, ${ }^{37}$ so the question of robustness with a quantitative readout is a meaningful one. (ii) While it is an autocatalytic, exponential amplification chemistry, its mechanism $^{38}$ is sufficiently complex that it was not obvious whether its initiation phase or propagation phase, and therefore the digital or kinetic format, would be more affected by perturbations. (iii) Digital LAMP has been recently demonstrated on various microfluidic platforms. ${ }^{36,39,40} \mathrm{We}$ used a microfluidic SlipChip device ${ }^{41}$ because it is well-suited for simple confinement and amplification of single molecules, ${ }^{42}$ it is convenient for performing multistep reactions on single molecules, ${ }^{36,43}$ and it has been validated with dRT-LAMP. ${ }^{36}$ We used a two-step RT-LAMP protocol because it is more efficient $^{36}$ than one-step RT-LAMP for the specific sequences used in this study. Also, RT-LAMP is an attractive amplification chemistry ${ }^{44}$ for LRS because it does not require thermocycling equipment and can be run using chemical heaters that do not require electricity. ${ }^{45,46}$ Furthermore, it is compatible with highly fluorescent calcein-based readout chemistry. ${ }^{47}$

First, we asked whether quantitative measurements by realtime RT-LAMP assays are robust to changes in temperature. We tested the robustness of a two-step real-time RT-LAMP assay to temperature fluctuations using a commercial instru- 

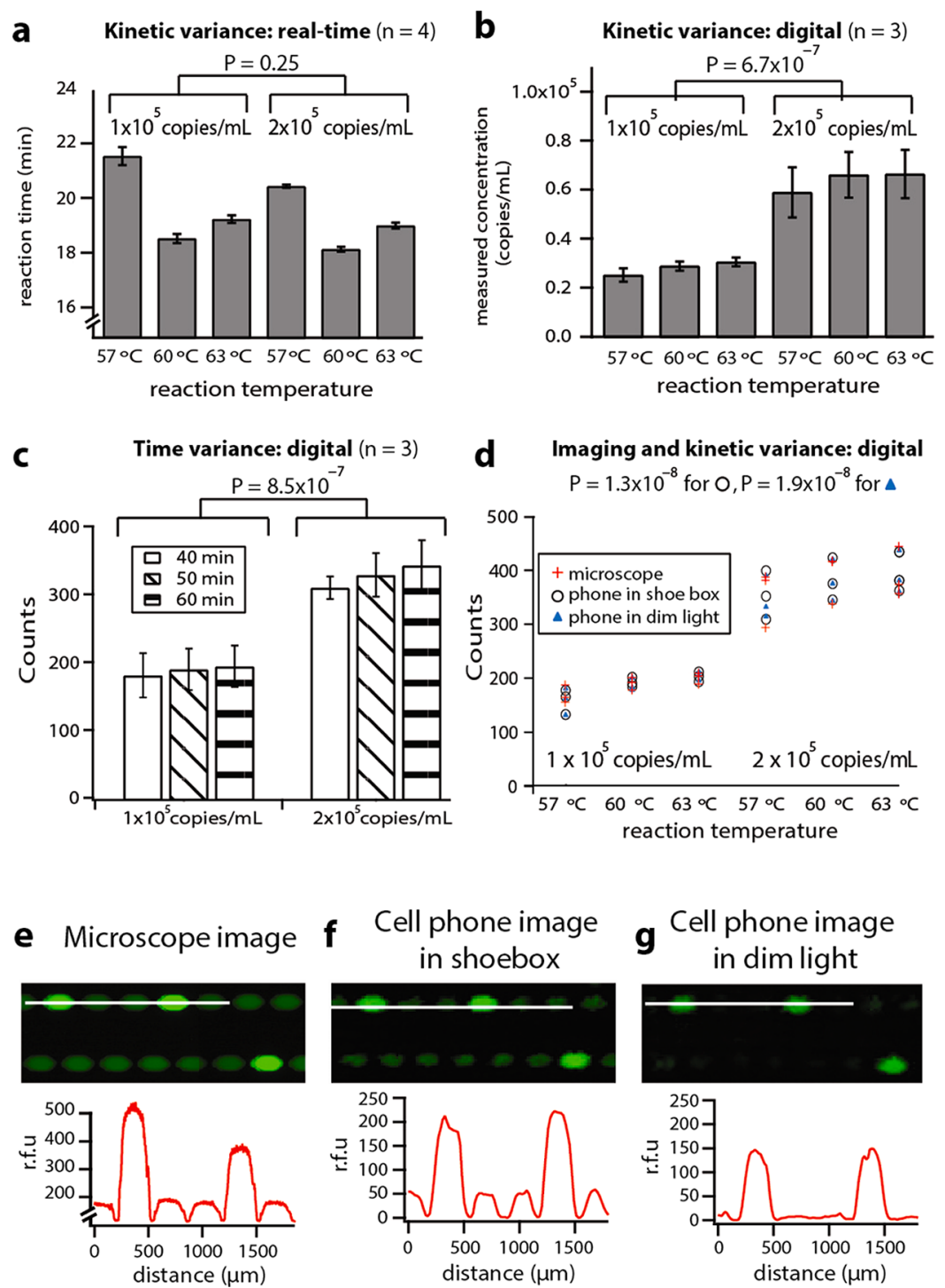

Figure 2. Evaluation of the robustness of real-time RT-LAMP versus digital RT-LAMP with respect to changes in temperature, time, and imaging conditions. (a, b) Graphs showing the results of (a) real-time RT-LAMP experiments and (b) digital RT-LAMP experiments for two concentrations across a 6-degree temperature range. Imaging was performed with a microscope. (c) A graph showing the number of positive counts from dRTLAMP experiments for two concentrations at various reaction times. (d) A plot comparing the data obtained from imaging with a microscope in part (b), data obtained from imaging dRT-LAMP with a cell phone in a shoebox, and data obtained from imaging dRT-LAMP in dim lighting $(\sim 3$ lux $)$ across a 6-degree temperature range. $P$-values denote statistical significance of all data for each concentration at a given imaging condition, irrespective of temperature (the null hypothesis being that the two concentrations were equivalent). (e-g) Top: Cropped and enlarged images of a dRT-LAMP reaction imaged with a microscope (e), a cell phone and shoe box ( $\mathrm{f}$ ), and a cell phone in dim lighting (g). Bottom: A corresponding line scan indicates fluorescence output from the region marked in white in each image. False color has been added in (e). The number of positives in each dRT-LAMP experiment imaged with a cell phone was counted manually. Error bars represent the standard deviation of the distribution.

ment (Figure 2a; see Supplementary Figure S1, Supporting Information, for one-step real-time RT-LAMP results). The precision of the assay for measuring two concentrations $(1 \times$ $10^{5}$ and $2 \times 10^{5}$ copies $\left./ \mathrm{mL}\right)$ of HIV-1 RNA at three temperatures over a 6-degree temperature range $(57,60,63$ ${ }^{\circ} \mathrm{C}$ ) was tested by comparing the reaction time (see Supporting Information online) for these two concentrations measured on an Eco real-time PCR machine. At each individual temperature, the real-time RT-LAMP assay could successfully distinguish between the two concentrations (at $57{ }^{\circ} \mathrm{C} p=0.007$, at $60{ }^{\circ} \mathrm{C} p$ $=0.01$, at $63{ }^{\circ} \mathrm{C} p=0.04$, the null hypothesis being that the two concentrations were identical). Therefore, we concluded that the assay itself was performing properly. The assay, however, was not robust to temperature fluctuations: changes of $3{ }^{\circ} \mathrm{C}$ introduced a larger change in the assay readout (reaction time) than the 2-fold change in the input concentration. Therefore, when temperature is not controlled precisely, this real-time RTLAMP assay cannot resolve a 2 -fold change in concentration of the input HIV-1 RNA.

We then tested whether a digital format of this RT-LAMP assay performed on a chip was robust to the same changes in temperature as those tested in the real-time RT-LAMP experiments (Figure 2b). For the dRT-LAMP experiments, the concentrations of HIV-1 RNA were determined by counting the number of positive wells on each chip after a 60 min reaction and then using Poisson statistics (detailed in the Supporting Information online). The dRT-LAMP assay could also distinguish between the two concentrations at each 
temperature (at $57^{\circ} \mathrm{C} p=0.03$, at $60^{\circ} \mathrm{C} p=0.02$, at $63{ }^{\circ} \mathrm{C} p=$ $0.02)$. In contrast to the real-time assay, the dRT-LAMP assay was robust to these temperature changes and resolved a 2-fold change in concentration despite these fluctuations $(p=7 \times$ $\left.10^{-7}\right)$. In these experiments, a Leica DMI-6000 microscope equipped with a Hamamatsu ORCA R-2 cooled CCD camera was used to image the dRT-LAMP devices. This setup provides an even illumination field and, therefore, intensity of the positive well was not a function of position (see Supporting Information online).

Next, we tested whether the dRT-LAMP assay was robust to variance in reaction time. We performed dRT-LAMP reactions with concentrations of $1 \times 10^{5}$ and $2 \times 10^{5}$ copies $/ \mathrm{mL}$ at a reaction temperature of $63{ }^{\circ} \mathrm{C}$ and imaged the reaction every minute using a Leica MZFLIII fluorescent stereomicroscope (detailed in the Supporting Information online). At each time point, the number of positive reactions was counted, and the results were averaged over three replicates (Figure 2c). For each of the two concentrations, we grouped together the raw counts at 40, 50, and 60 min-reaction times. Statistical analysis was used to reject the null hypothesis that these groups were the same $\left(p\right.$-value of $\left.8.5 \times 10^{-7}\right)$. Therefore, we conclude that the dRT-LAMP assay is robust to variance in reaction time, and an exact reaction time is not needed in the digital assay.

Next, we tested the robustness of the dRT-LAMP assay to poor imaging conditions using a Nokia 808 PureView cell phone with simple optical attachments (detailed in the Supporting Information online). The flash function of the cell phone was used to excite fluorescence through an excitation filter attached to the phone, and the camera of the cell phone was used to image fluorescence through an emission filter also attached to the cell phone. The results obtained with the cell phone were compared with those obtained with a microscope (Figure 2d). We tested the cell phone's imaging abilities under two lighting conditions: first, the dRT-LAMP assays were photographed in a shoe box, and second, in a dimly lit room with a single fluorescent task light in a corner. The light intensity at the point where the measurements were taken in the dimly lit room was $\sim 3$ lux as measured by an AEMC Instruments Model 810 light meter.

To evaluate whether imaging with a cell phone yields robust results, we performed statistical analysis of data obtained by cell phone imaging under each of the two lighting conditions. For imaging with a shoe box, we grouped all data obtained at the first concentration $\left(1 \times 10^{5}\right.$ copies $\left./ \mathrm{mL}\right)$ across all three temperatures into a first set, and all data obtained at the second concentration $\left(2 \times 10^{5}\right.$ copies $\left./ \mathrm{mL}\right)$ across all three temperatures into a second set. We then calculated a $p$-value of $1.3 \times$ $10^{-8}$ for the two sets (the null hypothesis being that the two concentrations were identical), which suggests that this imaging method could be used to differentiate between the two concentrations both at constant temperatures and even despite temperature changes. When we repeated this procedure for imaging in a dimly lit room, we calculated a $p$-value of $1.9 \times$ $10^{-8}$, indicating that the two concentrations could be distinguished with statistical significance in this scenario as well. Therefore, this dRT-LAMP assay was robust to the double perturbation of nonideal imaging conditions and temperature fluctuations.

We then tested whether other digital assays, such as digital PCR (dPCR), were sufficiently robust to poor imaging conditions to be analyzed with a cell phone. PCR amplification monitored with an intercalating dye such as Evagreen produces only a 2 - to 4 -fold change in fluorescence intensity as the reaction transitions from negative to positive. ${ }^{42}$ In our devices, the absolute intensity of fluorescence in the positive reaction in dPCR was approximately 6-15 times lower than that in dRTLAMP monitored with the calcein dye. When we conducted a dRT-PCR experiment using the same reaction volumes as those in the dRT-LAMP assays, we could easily distinguish positive from negative counts when the chip was imaged using a microscope, ${ }^{36}$ as expected. ${ }^{42}$ While it was also possible to image some of the dPCR chips using the cell phone, the signal was not sufficiently bright and robust for unambiguous analysis, and therefore, we did not pursue this direction further in this manuscript. We also tested the cell phone's ability to image the results on a spatially multiplexed PCR chip. ${ }^{48}$ This chip uses larger reaction volumes ( $78 \mathrm{~nL}$ as opposed to $6 \mathrm{~nL}$ ), thus enabling more fluorescent light to be emitted and collected per well. In this chip (Figure 3a,b), multiple primer pairs are

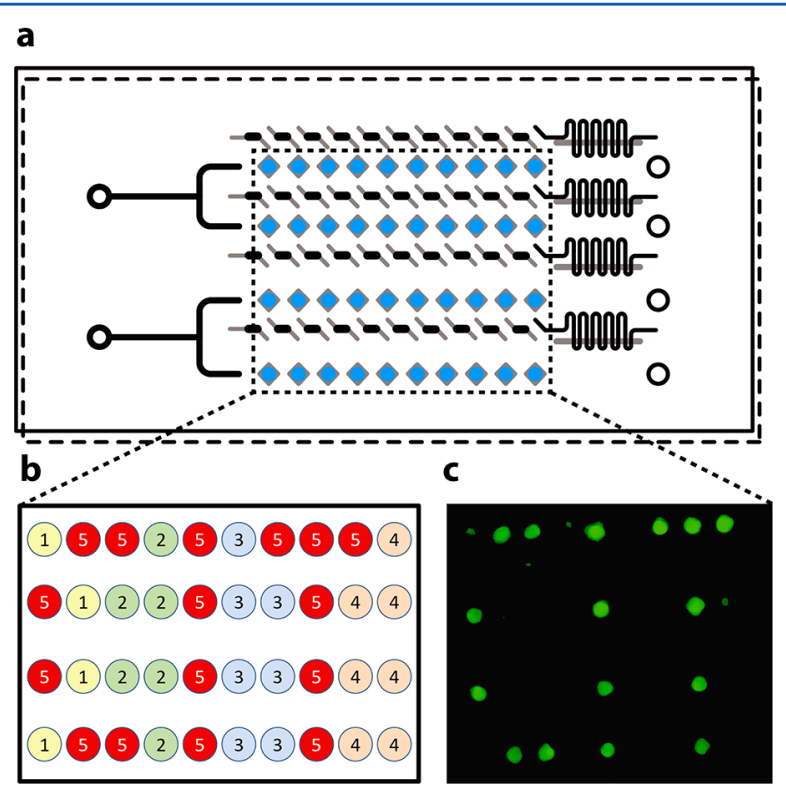

Figure 3. Cell phone imaging of multiplexed PCR on a SlipChip device using five different primer sets and a single template. (a) A schematic drawing of a SlipChip device that has been preloaded with primers. (b) A schematic drawing showing the arrangement of the five primer sets on the device: $1=E$. coli nlp gene, $2=P$. aeruginosa vic gene, $3=$ C. albicans calb gene, $4=$ Pseudomonas $16 \mathrm{~S}$, and $5=S$. aureus nuc gene; sequences are provided in Supplementary Table S1, Supporting Information. (c) A cell phone image of a SlipChip after loading it with $S$. aureus genomic DNA and performing PCR amplification. Wells containing the primer for S. aureus (green) increased in fluorescence to form the designed pattern. The intensity levels of the image have been adjusted, and the image has been smoothed to enhance printed visibility (details are included in the Supporting Information online).

preloaded into one set of wells, a sample is loaded into the second set of wells, and a "slip" combines the two sets of wells, thus enabling subsequent PCR amplification. Here, we used a five-plexed assay, in which one primer set was specific to the $S$. aureus genome (Figure $3 \mathrm{~b}$, detailed in the Supporting Information online). When $S$. aureus genomic DNA was loaded onto the device and the PCR reaction was performed, no nonspecific amplification was observed and a positive result was indicated by the appearance of the pattern on the device, as designed. This pattern, formed by PCR amplification in these 
larger wells, could be also visualized by the cell phone (Figure $3 c)$.

Finally, we tested whether this combination of dRT-LAMP chemistry and cell phone imaging was robust to automated processing of images and data analysis. When high-quality images, such as those taken with a microscope, are available, image processing and quantification of the positive signals can be performed simply by setting an intensity threshold and then counting the number of spots on the resulting image that exceed this threshold. For example, a threshold of 190 au was set for the data obtained with the microscope, and similar results were obtained by adjusting that threshold by as much as 150 units (Figure 4).

a Kinetic variance combined with variance of the data analysis: digital

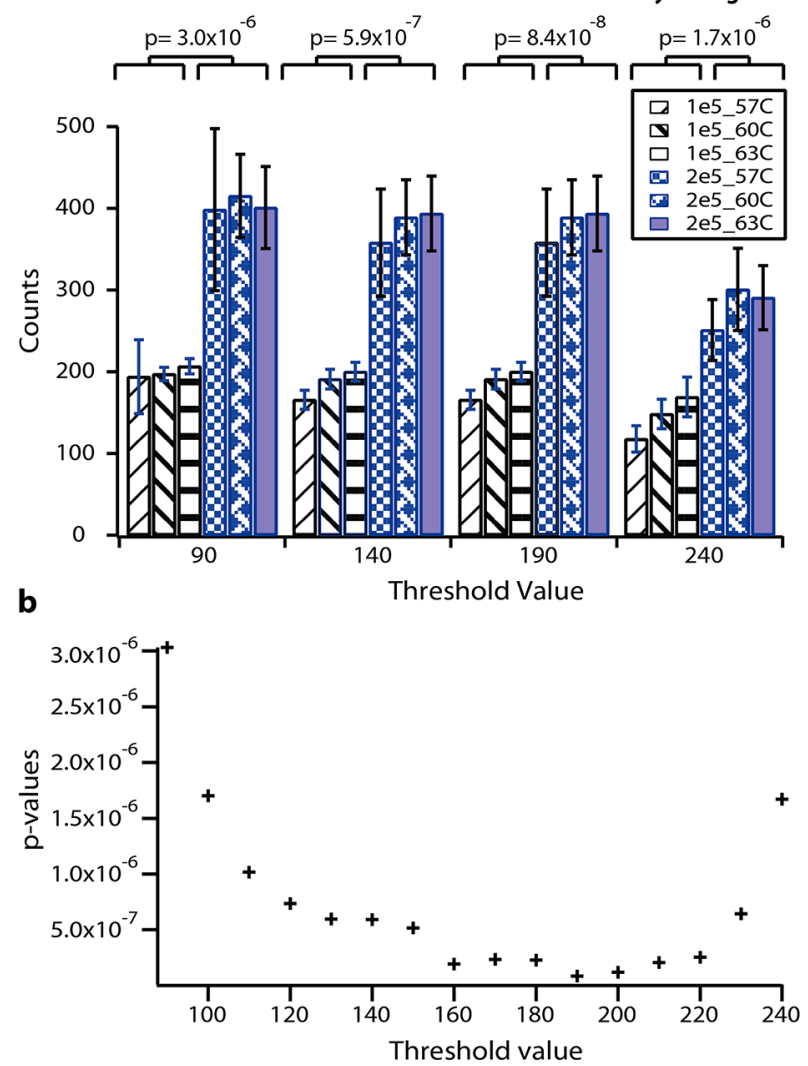

Figure 4. Robustness of digital dRT-LAMP imaged with a microscope to thresholding used to differentiate positive and negative wells. (a) A graph showing the number of positive reactions observed when imaging the dRT-LAMP reactions with a microscope as a function of the threshold value used to calculate the number of positives. Separation of the two data sets is easily observed even when changing the threshold value by 150 fluorescence units. (b) A plot of the $p$ values generated by comparing the two concentrations at threshold values between 90 and 240 . The minimum $p$-value is observed at a threshold of 190 .

This method is not, however, suitable for images taken with a cell phone for two reasons: (i) the short focal length $(6 \mathrm{~cm})$ creates significant variation in the illumination intensity of the flash (see Supplementary Figure S3, Supporting Information), and (ii) the imaging sensor has a much lower signal-to-noise ratio than those typically found in scientific instrumentation. Therefore, to test whether accurate automatic analysis of assays was possible, we wrote a custom image processing algorithm and implemented it in Labview software (detailed in the
Supporting Information online). Once an image was taken, it was automatically transferred to a remote server in "the cloud" (Figure $5 b$ ). The uploaded file was automatically analyzed by the server, and then the results were reported via email (Figure 5c). A video demonstrating that a minimally trained user can use the cell phone imaging approach described in this paper is available in the Supporting Information online (Video S1). We included error detection in the custom algorithm to ensure that the image included the device in its entirety (Figure $5 \mathrm{c}$ and Supporting Information online). This detection algorithm looked for four red circles on the device (Figure 5a), and if fewer than four were found, it generated an error message (Figure 5c, right). We tested the robustness of this cell phone imaging procedure to automated processing by directly comparing microscope image results quantified with Metamorph to cell phone images quantified with Labview over more than a hundred-fold concentration range (Figure 5d). A line of best fit of the compared data was found to have a slope of 0.968 and an $R^{2}$ value of 0.9997 , suggesting that this digital assay is robust to automated image processing even under poor imaging conditions and is suitable for use over a wide dynamic range.

We emphasize that, while we established robust automated counting of HIV-1 RNA molecules using digital amplification chemistry and a cell phone, this is only one part of the full diagnostic assay and several additional advances are needed for deployment under LRS. Simple chemical heaters ${ }^{45,46}$ that have been shown to work with RT-LAMP in qualitative assays would need to be incorporated into a digital platform. LRS-suitable techniques for sample preparation would need to be incorporated as well, as standard laboratory procedures were used for these experiments. The robustness of dRT-LAMP to factors such as sequence diversity of viruses present in clinical samples, changes in activity of reagents during storage, and quality of sample preparation remains to be tested. While offloading the analysis of images to "the cloud" provides a number of benefits, including traceability and archiving of raw data, global access, and compatibility with virtually all smartphone operating systems, it requires a wireless data connection of sufficiently high bandwidth; thus, direct onphone analysis could be preferable in some scenarios. As cell phone technology evolves at a rapid pace and smartphone operating systems converge with classic point-and-shoot cameras, advanced imaging sensors and excitation sources could offer additional opportunities for robust imaging of other amplification assays in LRS. In addition, for this study, we have used lab-produced glass microfluidic chips; under LRS, massproduced variants of this device would need to be used.

\section{CONCLUSIONS}

We have shown that HIV-1 RNA amplification using RTLAMP assays in the traditional real-time format is, as expected, not robust to temperature fluctuations. In contrast, digital counting of HIV-1 RNA molecules using dRT-LAMP is robust not only to temperature fluctuations but also to changes in reaction time, artifacts introduced by poor-quality cell phone imaging, and automatic analysis. Bright fluorescent output with a high ratio of positive to negative signals of the digital LAMP chemistry was necessary to observe robustness to imaging and automated analysis. Below, we point out several implications of these findings.

Given the complexity of the mechanism of the LAMP amplification reaction, ${ }^{38}$ we were surprised to find it was robust, in the digital format, to changes in temperature and time. This 
a Image device with a cell phone

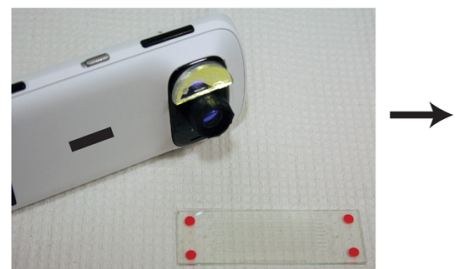

C Automatically receive results via email
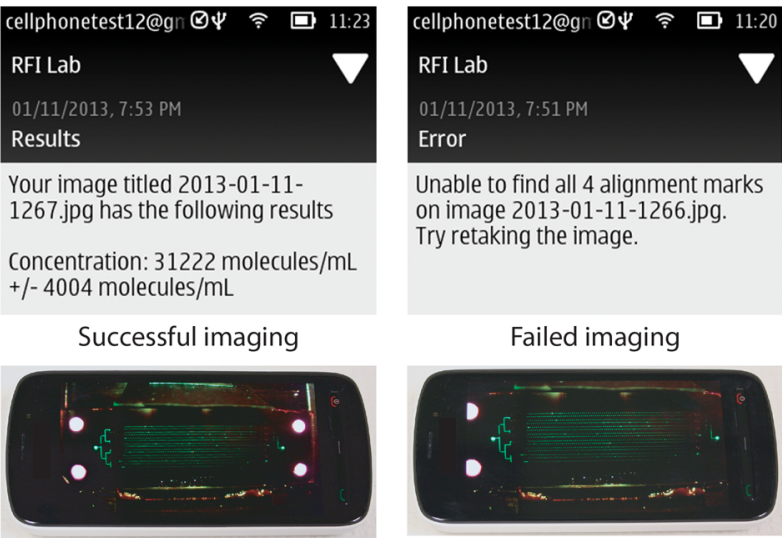

Unable to find all 4 alignment marks on image 2013-01-11-1266.jpg.

Try retaking the image.

Failed imaging

d Automatic analysis robust over 100-fold dynamic range
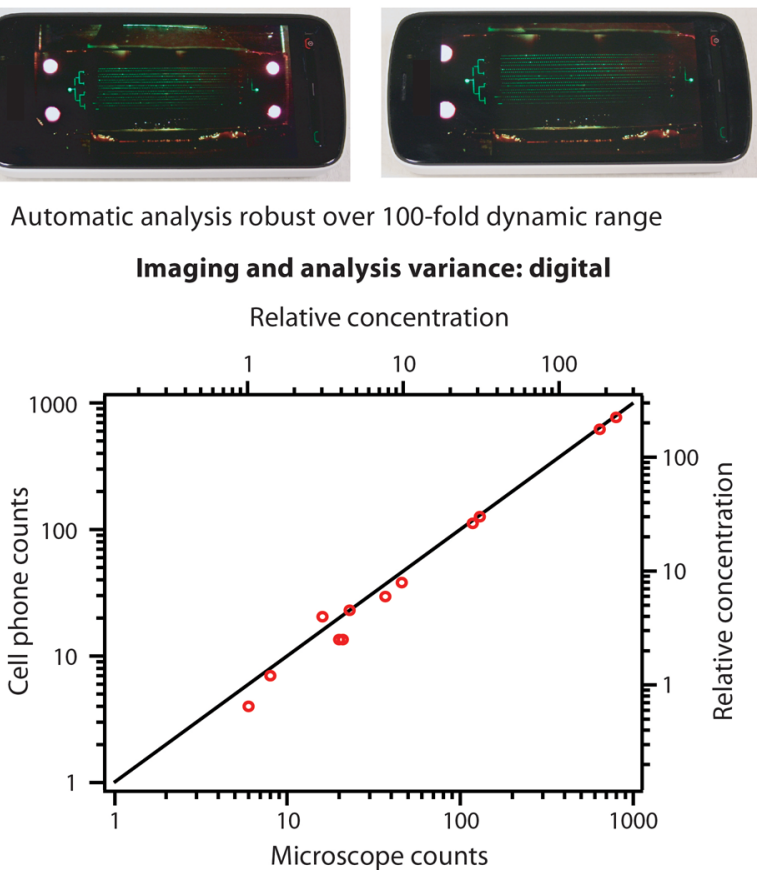

Figure 5. The image analysis workflow used to count molecules via digital amplification with a SlipChip and a cell phone. Nokia logo has been redacted per Journal policy. (a) Left: A cell phone with objective for detecting fluorescence. Right: A device labeled with four red circles that the imaging processing algorithm uses to confirm that the entire device has been imaged. (b) A cartoon representing a cloud-based server that analyzes photographs taken by the user, archives the raw data, and sends the results to the appropriate party. (c) Top: Screenshots of a cell phone screen showing email messages received by a prespecified recipient after analysis of successful (left) and unsuccessful (right) imaging. Bottom: the images that were analyzed in each case. (d) A graph comparing the raw positive counts from a cell phone processed automatically by Labview software ( $y$-axis) to imaging and thresholding performed with an epifluorescence microscope ( $x$-axis) using a more than 100 -fold concentration change. A video demonstrating that a minimally trained user can use the cell phone imaging approach described in this paper is available in the Supporting Information online (Video S1).

result suggests that the probability of initiation of the amplification cascade from a single molecule does not significantly change and this initiation is not delayed with changes in temperature, despite temperature-dependent changes in the equilibria of DNA-DNA hybridization, enzyme-DNA binding, and changes in the rates of the various enzymatic steps of the reaction. We hypothesize that as long as the efficiency of the initiation step is high and is not strongly affected by the perturbation of interest, robustness to this perturbation should be a general property of digital amplification schemes. We are interested in a future direct comparison of robustness of real-time and digital recombinase polymerase amplification (RPA $)^{43}$ to temperature, time, and imaging perturbations. Additional experimental studies are needed to test whether other nonlinear and autocatalytic amplification systems ${ }^{1-12}$ satisfy this criterion and show robustness in the digital format to changes in temperature, time, and other experimental perturbations. SlipChip devices are attractive for such studies because they support multistep manipulations of single molecules ${ }^{36,43}$ and can be made in glass to be compatible with a wide range of chemistries. In general, such studies could be performed using any microfluidic platforms that support digital single-molecule manipulations. Theoretical studies are also needed to predict the level of robustness of specific amplification reactions and also to predict more generally which reaction architectures are likely to be robust to which perturbations, leading to the design of new amplification chemistries. Robustness of biological systems, e.g., robustness of circadian clocks to temperature fluctuations, ${ }^{16}$ may provide an inspiration for such studies.

If these experimental and theoretical studies are successful, we may see an emergence of nonlinear amplification schemes that are especially suitable for quantitative measurements under LRS because they are ultrarapid and specific, provide bright positive and dim negative signals, and are robust to experimental perturbations. Finally, we share the analogy that motivates our work to explore the robustness of quantitative measurements and their applicability to LRS. Many countries in the developing world recently underwent a technological revolution. Their growth had been hindered by lack of a communication infrastructure, since replicating the land-line based model of the developed world would have been impractical and prohibitively expensive. The revolution happened when these countries bypassed the land-line paradigm and leapfrogged directly to wireless technologies. An analogous technological transformation may occur for quantitative molecular measurements under LRS without the need to create the infrastructure required to carefully control and analyze kinetic assays. Instead, studies of robustness of chemical amplification and signal transduction schemes may allow the quantitative molecular measurements and diagnostics in the developing world, the field, and the home to leapfrog directly to the more robust digital, single-molecule approaches.

\section{ASSOCIATED CONTENT}

\section{Supporting Information}

Supporting information and a supplementary video. This material is available free of charge via the Internet at http:// pubs.acs.org.

\section{AUTHOR INFORMATION}

\section{Corresponding Author}

*E-mail: rustem.admin@caltech.edu. Fax: 626-568-8743. 


\section{Author Contributions}

${ }^{I}$ D.A.S., M.A.K., and B.S. contributed equally to this work.

\section{Notes}

The authors declare the following competing financial interest(s): R.F.I. has a financial interest in SlipChip Corp.

\section{ACKNOWLEDGMENTS}

This work was supported in part by DARPA Cooperative Agreement No. HR0011-11-2-0006, NIH grant No. R01EB012946, and NIH grant No. 5DP1OD003584. We thank Whitney Robles for contributions to writing and editing this manuscript. We also thank the five-year-old volunteer for performing the demonstration shown in Video S1.

\section{REFERENCES}

(1) Baker, M. S.; Phillips, S. T. J. Am. Chem. Soc. 2011, 133, 51705173

(2) Gerdts, C. J.; Sharoyan, D. E.; Ismagilov, R. F. J. Am. Chem. Soc. 2004, 126, 6327-6331.

(3) Jiang, Y.; Li, B.; Milligan, J. N.; Bhadra, S.; Ellington, A. D. J. Am. Chem. Soc. 2013, 135, 7430-7433.

(4) Kottani, R; Majjigapu, J. R; Kurchan, A.; Majjigapu, K.; Gustafson, T. P.; Kutateladze, A. G. J. Am. Chem. Soc. 2006, 128, 14794-14795.

(5) Mohapatra, H.; Schmid, K. M.; Phillips, S. T. Chem. Commun. 2012, 48, 3018-3020.

(6) Olea, C.; Horning, D. P.; Joyce, G. F. J. Am. Chem. Soc. 2012, 134, $8050-8053$.

(7) Taton, T. A.; Mirkin, C. A.; Letsinger, R. L. Science 2000, 289, $1757-1760$

(8) Tian, L.; Weizmann, Y. J. Am. Chem. Soc. 2012, 135, 1661-1664.

(9) Zuo, X.; Xia, F.; Xiao, Y.; Plaxco, K. W. J. Am. Chem. Soc. 2010, 132, 1816-1818.

(10) Bánsági, T.; Vanag, V. K.; Epstein, I. R. Science 2011, 331, 1309-1312.

(11) Epstein, I. R.; Pojman, J. A. An Introduction to Nonlinear Chemical Dynamics: Oscillations, Waves, Patterns, and Chaos; Oxford University Press: New York, 1998.

(12) Qian, L.; Winfree, E. Science 2011, 332, 1196-1201.

(13) Alon, U.; Surette, M. G.; Barkai, N.; Leibler, S. Nature 1999, $397,168-171$.

(14) Kitano, H. Mol. Syst. Biol. 2007, 3, 137.

(15) Lucchetta, E. M.; Lee, J. H.; Fu, L. A.; Patel, N. H.; Ismagilov, R. F. Nature 2005, 434, 1134-1138.

(16) Buhr, E. D.; Yoo, S.-H.; Takahashi, J. S. Science 2010, 330, 379385.

(17) Girones, R.; Ferrus, M. A.; Alonso, J. L.; Rodriguez-Manzano, J.; Calgua, B.; Correa, A. D.; Hundesa, A.; Carratala, A.; Bofill-Mas, S. Water Res. 2010, 44, 4325-4339.

(18) Postollec, F.; Falentin, H.; Pavan, S.; Combrisson, J.; Sohier, D. Food Microbiol. 2011, 28, 848-861.

(19) The FEM Microbiology Action Team. Method Validation of U.S. Environmental Protection Agency Microbiological Methods of Analysis, 2009; http://www.epa.gov/fem/pdfs/final_microbiology_method_ guidance 110409.pdf (accessed June 19, 2013).

(20) West, J. S.; Atkins, S. D.; Emberlin, J.; Fitt, B. D. L. Trends Microbiol. 2008, 16, 380-387.

(21) Heid, C. A.; Stevens, J.; Livak, K. J.; Williams, P. M. Genome Res. 1996, 6, 986-994.

(22) Belgrader, P.; Benett, W.; Hadley, D.; Richards, J.; Stratton, P.; Mariella, R., Jr.; Milanovich, F. Science (Washington) 1999, 284, 449450.

(23) Neuzil, P.; Zhang, C.; Pipper, J.; Oh, S.; Zhuo, L. Nucleic Acids Res. 2006, 34, e77-e77.

(24) Yager, P.; Edwards, T.; Fu, E.; Helton, K.; Nelson, K.; Tam, M. R.; Weigl, B. H. Nature 2006, 442, 412-418.
(25) Calmy, A.; Ford, N.; Hirschel, B.; Reynolds, S. J.; Lynen, L.; Goemaere, E.; de la Vega, F. G.; Perrin, L.; Rodriguez, W. Clin. Infect. Dis. 2007, 44, 128-134.

(26) Roche Diagnostic Systems, Inc. AMPLICOR HIV-1 MONITORTM TEST Standard/UltraSensitive. http://www.fda.gov/ downloads/BiologicsBloodVaccines / BloodBloodProducts / ApprovedProducts/PremarketApprovalsPMAs/UCM093317.pdf (accessed June 19, 2013).

(27) Panel on Antiretroviral Guidelines for Adults and Adolescents. Guidelines for the use of antiretroviral agents in HIV-1-infected adults and adolescents; Department of Health and Human Services: Bethesda, MD, 2013; Vol. 2013; http://aidsinfo.nih.gov/ContentFiles/ AdultandAdolescentGL.pdf (accessed June 19, 2013).

(28) Sykes, P.; Neoh, S.; Brisco, M.; Hughes, E.; Condon, J.; Morley, A. BioTechniques 1992, 13, 444.

(29) Ottesen, E. A.; Hong, J. W.; Quake, S. R.; Leadbetter, J. R. Science 2006, 314, 1464-1467.

(30) Rissin, D. M.; Walt, D. R. J. Am. Chem. Soc. 2006, 128, 62866287.

(31) Kreutz, J. E.; Munson, T.; Huynh, T.; Shen, F.; Du, W.; Ismagilov, R. F. Anal. Chem. 2011, 83, 8158-8168.

(32) Martinez, A. W.; Phillips, S. T.; Carrilho, E.; Thomas, S. W.; Sindi, H.; Whitesides, G. M. Anal. Chem. 2008, 80, 3699-3707.

(33) Breslauer, D. N.; Maamari, R. N.; Switz, N. A.; Lam, W. A.; Fletcher, D. A. PLoS One 2009, 4, No. e6320.

(34) Zhu, H. Y.; Isikman, S. O.; Mudanyali, O.; Greenbaum, A.; Ozcan, A. Lab Chip 2013, 13, 51-67.

(35) Bustin, S. A.; Benes, V.; Garson, J. A.; Hellemans, J.; Huggett, J.; Kubista, M.; Mueller, R.; Nolan, T.; Pfaffl, M. W.; Shipley, G. L. Clin. Chem. 2009, 55, 611-622.

(36) Sun, B.; Shen, F.; McCalla, S. E.; Kreutz, J. E.; Karymov, M. A.; Ismagilov, R. F. Anal. Chem. 2013, 85, 1540-1546.

(37) Francois, P.; Tangomo, M.; Hibbs, J.; Bonetti, E.-J.; Boehme, C. C.; Notomi, T.; Perkins, M. D.; Schrenzel, J. FEMS Immunol. Med. Microbiol. 2011, 62, 41-48.

(38) Nagamine, K.; Hase, T.; Notomi, T. Mol. Cell. Probes 2002, 16, 223-229.

(39) Gansen, A.; Herrick, A. M.; Dimov, I. K.; Lee, L. P.; Chiu, D. T. Lab Chip 2012, 12, 2247-2254.

(40) Zhu, Q. Y.; Gao, Y. B.; Yu, B. W.; Ren, H.; Qiu, L.; Han, S. H.; Jin, W.; Jin, Q. H.; Mu, Y. Lab Chip 2012, 12, 4755-4763.

(41) Du, W.; Li, L.; Nichols, K. P.; Ismagilov, R. F. Lab Chip 2009, 9, 2286-2292.

(42) Shen, F.; Du, W.; Kreutz, J. E.; Fok, A.; Ismagilov, R. F. Lab Chip 2010, 10, 2666-2672.

(43) Shen, F.; Davydova, E. K.; Du, W.; Kreutz, J. E.; Piepenburg, O.; Ismagilov, R. F. Anal. Chem. 2011, 83, 3533-3540.

(44) Curtis, K. A.; Rudolph, D. L.; Owen, S. M. J. Virol. Methods 2008, 151, 264-270.

(45) Liu, C. C.; Mauk, M. G.; Hart, R.; Qiu, X. B.; Bau, H. H. Lab Chip 2011, 11, 2686-2692.

(46) Curtis, K. A.; Rudolph, D. L.; Nejad, I.; Singleton, J.; Beddoe, A.; Weigl, B.; LaBarre, P.; Owen, S. M. PLoS One 2012, 7, No. e31432.

(47) Tomita, N.; Mori, Y.; Kanda, H.; Notomi, T. Nat. Protoc. 2008, $3,877-882$.

(48) Shen, F.; Du, W.; Davydova, E. K.; Karymov, M. A.; Pandey, J.; Ismagilov, R. F. Anal. Chem. 2010, 82, 4606-4612. 